\title{
An environmental benign process for cobalt and lithium recovery from spent lithium-ion batteries by mechanochemical approach
}

\author{
Meng-Meng Wang, Cong-Cong Zhang, Fu-Shen Zhang* \\ Research Center for Eco-environmental Sciences, Chinese Academy of Sciences, 18 Shuangqing Road, Beijing 100085, China
}

\section{A R T I C L E I N F O}

\section{Article history:}

Received 28 December 2015

Revised 29 February 2016

Accepted 2 March 2016

Available online 7 March 2016

\section{Keywords:}

Spent lithium-ion batteries

Mechanochemical approach

Lithium cobalt oxide

EDTA

Metal recovery

\begin{abstract}
A B S T R A C T
In the current study, an environmental benign process namely mechanochemical approach was developed for cobalt and lithium recovery from spent lithium-ion batteries (LIBs). The main merit of the process was that neither corrosive acid nor strong oxidant was applied. In the proposed process, lithium cobalt oxide (obtained from spent LIBs) was firstly co-grinded with various additives in a hermetic ball milling system, then Co and Li could be easily recovered by a water leaching procedure. It was found that EDTA was the most suitable co-grinding reagent, and $98 \%$ of Co and $99 \%$ of Li were respectively recovered under optimum conditions: $\mathrm{LiCoO}_{2}$ to EDTA mass ratio $1: 4$, milling time $4 \mathrm{~h}$, rotary speed $600 \mathrm{r} / \mathrm{min}$ and ball-to-powder mass ratio 80:1, respectively. Mechanisms study implied that lone pair electrons provided by two nitrogen atoms and four hydroxyl oxygen atoms of EDTA could enter the empty orbit of Co and Li by solid-solid reaction, thus forming stable and water-soluble metal chelates Li-EDTA and Co-EDTA. Moreover, the separation of $\mathrm{Co}$ and Li could be achieved through a chemical precipitation approach. This study provides a high efficiency and environmentally friendly process for Co and Li recovery from spent LIBs.
\end{abstract}

(c) 2016 Elsevier Ltd. All rights reserved.

\section{Introduction}

Environmental pollution and energy crisis have driven the progressing of new energy vehicles and the development of power batteries (Kang et al., 2013). Compared with lead-acid batteries and nickel metal hydride, lithium-ion batteries (LIBs) are expected to dominate the market in terms of their high working-voltage, large capacity, long circle-life and non-memory effect, especially with the rise of plug-in hybrid and purely electrically driven battery electric vehicles (Majeau-Bettez et al., 2011; Notter et al., 2010; Scrosati and Garche, 2010). Recently, with the rapid upgrade and replacement of new energy vehicle, as well as electronic devices, huge amounts of spent LIBs are generated worldwide without any proper disposal. Take China for example, the total quantity and weight of discarded LIBs were estimated to reach 25 billion units up to 500 thousand tons by 2020 (Zeng et al., 2012). Generally, spent LIBs are composed of cathode, anode, electrolyte and separator, and the most widely used cathode material is lithium cobalt oxide $\left(\mathrm{LiCoO}_{2}\right)$, which is characterized by high specific energy density and durability (Scrosati and Garche, 2010). In view of the growing interest in environmental protection and resources sustainable use, recovery of spent LIBs especially

\footnotetext{
* Corresponding author.

E-mail address: fszhang@rcees.ac.cn (F.-S. Zhang).
}

$\mathrm{LiCoO}_{2}$ is becoming increasingly important, as it will largely help to alleviate the potential environmental pressures and solve the crisis of cobalt shortage.

Authorities have enforced regulations on spent batteries' disposal. In 2006, the European Parliament and the EU Council of Ministers revised the 1991 Battery Directive 91/157/EEC (Directive, 1991 ) covering batteries and accumulators. Since 2008 the new Battery Directive 2006/66/EC (Directive, 2006, 2008) prescribes the currently valid collecting targets and recycling efficiencies. The member states are obliged to reach a minimum collection rate for spent batteries and accumulators of $25 \%$ by 2012 and of $45 \%$ by 2016. Furthermore, Li-ion battery recycling processes will be obliged to reach a minimum recycling efficiency of $50 \%$ by average weight. A huge number of researches going on with respect to recycling processes, as comprehensively reviewed by Xu et al. (2008) and Zeng et al. (2014). The major drawback is that, the amount of spent batteries available for recycling is small and does not match the large number of secondary cells produced for every year. For example, 97 tons of spent NiMH batteries were recycled in Germany in 2003 which represents only 3\% of NiMH batteries produced in that year (Al-Thyabat et al., 2013).

Thus far, most researches on spent LIBs generally have been focused on valuable metals recovery. The main methods for metal recovery comprise physical dismantling (Bertuol et al., 2015; Zhang et al., 2013), metal leaching (Chen et al., 2011; Paulino 
et al., 2008; Sun and Qiu, 2011) and the separation of Co and Li (Joulié et al., 2014; Provazi et al., 2011; Wang et al., 2009). Among these studies, leaching of $\mathrm{Co}$ and $\mathrm{Li}$ from $\mathrm{LiCoO}_{2}$ powder using hydrometallurgical techniques had attracted wide attention, besides inorganic acids (Chen et al., 2015; Jha et al., 2013), organic oxalate (Sun and Qiu, 2012), organic citric acid (Li et al., 2010a), succinic acid (Li et al., 2015), oxalic acid (Zeng et al., 2015), tartaric acid and ascorbic acid (Nayaka et al., 2016) were used as leaching agents with satisfactory achievements. However, these acids could inevitably cause corrosion and liquor waste. Hence, economical, highly effective and environmentally friendly processes for recovery of Co and Li from spent LIBs are urgently desired.

In recent decades, considerable researches have been focused on metal recovery by mechanochemical method (Tan and Li, 2015; Yuan et al., 2012). With a non-thermal process, Kano et al. (2009) recovered indium (In) through mechanochemical reduction of $\mathrm{In}_{2} \mathrm{O}_{3} / \mathrm{ITO}$ by milling with $\mathrm{Li}_{3} \mathrm{~N}$ under a non-oxidative state of $\mathrm{NH}_{3}$ and/or $\mathrm{N}_{2}$ gas environment. Lee et al. (2013) recovered In from spent liquid crystal display (LCD) panels assisted with high energy ball milling and acid leaching. Shibata et al. $(2011,2012)$ used a mechanochemical method to recover tungsten and cobalt from tungsten carbide tool wastes. Saeki et al. (2004) and Zhang et al. (2007) developed a process for metal recovery from alloy-wastes and $\mathrm{LiCoO}_{2}$ powder via co-grinding with polyvinyl chloride (PVC). Compared with hydrometallurgical processes, these studies revealed that mechanochemical processes could not only obviously simplify metal leaching, but also avoid the generation of liquor waste in point of solid-solid reaction. Meanwhile, more than 99\% lead was extracted from spent lead-glass by a mechanochemical method when ethylenediaminetetraacetic acid disodium salt ( $\mathrm{Na}_{2}$ EDTA) was used as metal chelate reagent (Sasai et al., 2008). Furthermore, EDTA had been applied as metal-ion chelation in a process for separating metals in a mixed solution of Co and $\mathrm{Li}$ (Iizuka et al., 2013) and separating nickel from cobalt solutions (Chaudhary et al., 2000). The study implied that $\mathrm{Na}_{2}$ EDTA and EDTA are important chelating reagents which could form stable metal complexes with various metals.

In the present study, a novel mechanochemistry process with low energy consumption and high efficiency was developed for the recovery of $\mathrm{Co}$ and $\mathrm{Li}$ from spent LIBs. EDTA and $\mathrm{Na}_{2}$ EDTA were tentatively used as co-grinding reagents to chelate Co and Li from $\mathrm{LiCoO}_{2}$ powder. Various operating parameters were optimized and the mechanochemical residues were characterized. Furthermore, the reaction mechanisms of $\mathrm{LiCoO}_{2}$ co-grinding with different additives were discussed in detail and efficient approaches for Co and Li separation were established as well.

\section{Experimental}

\subsection{Materials and methods}

Spent LIBs were supplied by Beijing HuaXing Environmental Protection Co. Ltd, China. The LIBs were firstly discharged completely to avoid short-circuit or self-ignition during dismantling. The metallic shell, cathode material, anode material and organic separators were separated manually using a plier and a screwdriver. Then the cathode materials were crushed with a small high-speed universal pulverizer to separate aluminum film and $\mathrm{LiCoO}_{2}$ powder. Contents of $\mathrm{Co}$ and $\mathrm{Li}$ in $\mathrm{LiCoO}_{2}$ powder were measured by inductively coupled plasma optical emission spectrometer (ICP-OES, Prodigy, Leeman, USA) after digestion with aqua regia. Results indicated that $\mathrm{Co}$ and $\mathrm{Li}$ accounted for $51.8 \%$ and $6.5 \%$ of the total cathode materials (wt\%). All chemical reagents were of analytical grade and purchased from Chemical Reagent Company of Beijing.

\subsection{Analytical methods}

The concentrations of $\mathrm{Co}$ and $\mathrm{Li}$ in leaching liquor were measured by ICP-OES (ICP-OES, Prodigy, Leeman, USA). The crystal structure and surface morphology of the original and ball milled samples were characterized by X-ray diffraction (XRD, Philips PW 1700) using $\mathrm{Cu} \mathrm{K} \alpha$ radiation $(\gamma=1.5418 \AA$ ) with $30 \mathrm{kV}$ voltage and $30 \mathrm{~mA}$ current. The analysis of XRD data were carried out by MDI Jade 6.0 software.

\subsection{Recovery procedure}

The schematic diagram of Co and Li recovery from spent LIBs is shown in Fig. 1. All mechanochemical experiments were carried out in a planetary ball mill (QM-3SP2J) comprised of four $50 \mathrm{~mL}$ zirconia pots with zirconia balls as grinding medium. First, an appropriate proportion of $\mathrm{LiCoO}_{2}$ powder and co-grinding reagents, together with zirconia balls were sealed in the zirconia pots. Then the powder mixtures were ball milled at different rotary speed for different periods of time. The milled products and zirconia balls adherent with powder were then rinsed with $100 \mathrm{~mL}$ of deionized water and agitated for $30 \mathrm{~min}$. The leaching solution and residues were separated by vacuum filtration. In the subsequent chemical precipitation process, $\mathrm{Co}$ and $\mathrm{Li}$ in the leaching liquor were separated and recycled by addition of $\mathrm{NaOH}$ and $\mathrm{Na}_{2} \mathrm{CO}_{3}$, respectively. Cobalt oxides were first obtained after calcination of the precipitate at $500{ }^{\circ} \mathrm{C}$ for $2 \mathrm{~h}$, and then lithium carbonate was recovered after recrystallization and drying. The recovery rates of Co and $\mathrm{Li}$, determined by ICP-OES, were expressed in percentage by the following formula:

$\mathrm{W}=\frac{\mathrm{C} \cdot \mathrm{V}}{\mathrm{C}_{0} \cdot \mathrm{V}_{0}} \times 100 \%$

where $\mathrm{W}$ is the recovery rate; $\mathrm{C}_{0}$ and $\mathrm{C}$ are metal concentrations in solution before and after mechanochemical treatment; $V_{0}$ and $V$ are volumes of leaching liquor before and after mechanochemical treatment.

\section{Results and discussion}

\subsection{Screening of co-grinding reagents}

Five types of chlorides, including PVC, $\mathrm{NaCl}, \mathrm{NH}_{4} \mathrm{Cl}, \mathrm{ZnCl}_{2}$ and $\mathrm{FeCl}_{3}$, were tested as co-grinding reagents to supply exogenous

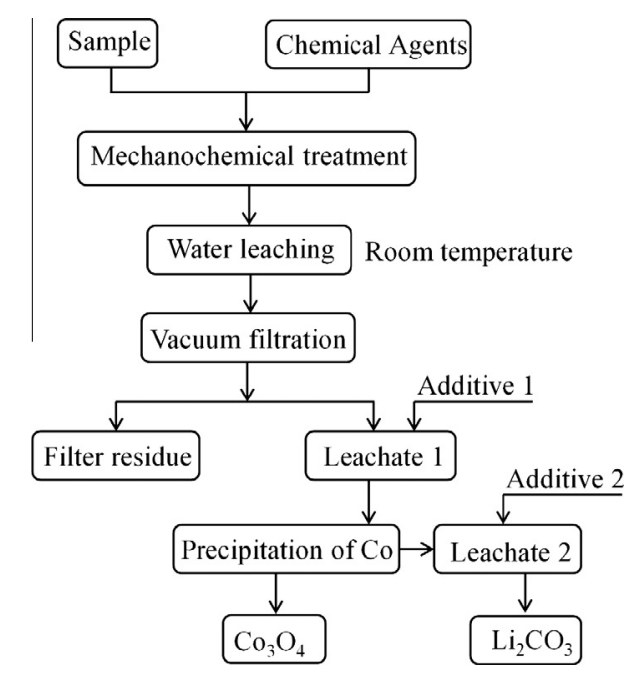

Fig. 1. Schematic diagram for Co and Li recovery from spent LIBs. 
chlorides. As can be seen in Fig. 2, Co and Li could be leached out to a certain degree from $\mathrm{LiCoO}_{2}$ after co-grinded with different additives, indicating that $\mathrm{Co}$ and $\mathrm{Li}$ inside $\mathrm{LiCoO}_{2}$ powder were tentatively converted into their chloride forms during mechanochemical process. It has been reported that mechanical forces such as shear, impact and squeezing exerted by ball milling, will transmit energy to powder, diminished powder particles and destroy the crystal structures (James et al., 2012; Nasser and Mingelgrin, 2012). Moreover, the ball milling process can reduce specific surface energy of reaction products so as to promote the impossible solid-solid reaction and enhance the reaction efficiency (Friščić, 2010).

However, as showed in Fig. 2, only a small amount of Li was leached out when PVC was co-grinded with $\mathrm{LiCoO}_{2}$, indicating that PVC, the polar polymer with amorphous structure, is not suitable to co-grind with $\mathrm{LiCoO}_{2}$, which is a crystal structure compound. During the co-grinding of $\mathrm{NaCl}$ with $\mathrm{LiCoO}_{2}$ powder, about $30 \%$ of $\mathrm{Li}$ and $\mathrm{Cl}$ were converted into water-soluble $\mathrm{LiCl}$, but $\mathrm{Co}$ was not converted into chloride form since the ionic bond of $\mathrm{NaCl}$ (bond energy $412 \mathrm{~kJ} \mathrm{~mol}^{-1}$ ) was broken by the mechanical force, thus $\mathrm{Li}$ in $\mathrm{LiCoO}_{2}$ with reduced atom radius and improved polarization ability would first bind with $\mathrm{Cl}$ to form $\mathrm{LiCl}$, while Co with lower reactivity could not form water-soluble compound (Zhang et al., 2007). Both Co and Li were leached out during ball milling between $\mathrm{NH}_{4} \mathrm{Cl}$ and $\mathrm{LiCoO}_{2}$. This could be attributed to the fact that $\mathrm{NH}_{4} \mathrm{Cl}$ was partially decomposed due to the external mechanical force, thus active $\mathrm{Cl}$ could react with $\mathrm{Li}$ and $\mathrm{Co}$ by solid-solid reaction. The chlorine donor $\mathrm{FeCl}_{3}$ finitely improved the recovery rates of both $\mathrm{Co}$ and $\mathrm{Li}$ compared with $\mathrm{NH}_{4} \mathrm{Cl}$ or $\mathrm{NaCl}$. As a covalent compound and strong acid salt, $\mathrm{FeCl}_{3}$ is more reactive than $\mathrm{NaCl}$, the ionic compound. It is more likely to react with $\mathrm{LiCoO}_{2}$ crystals whose surface activation energy was reduced during ball milling process. $\mathrm{FeCl}_{3}$ has the ability to convert $\mathrm{Li}$ and bind with loweractivity Co to form water-soluble ions. However, the recovery rates were generally low when $\mathrm{LiCoO}_{2}$ was co-grinded with the above chlorides since the ball milling system could not provide enough energy to break the chemical bonds of chlorides (Courtney and Dahn, 1997; Wang et al., 1999). Therefore, it is necessary to screen out more efficient co-grinding agents in view of the actual recovery effect and economic benefits.

Further experiments showed that, $71 \%$ of $\mathrm{Co}$ and $74 \%$ of $\mathrm{Li}$ were recovered when $\mathrm{LiCoO}_{2}$ was co-grinded with $\mathrm{Na}_{2}$ EDTA powder, and $98 \%$ of $\mathrm{Co}$ and $99 \%$ of $\mathrm{Li}$ were recycled when EDTA- $\mathrm{LiCoO}_{2}$ ball milling system was applied. $\mathrm{Na}_{2}$ EDTA and EDTA are all excellent metal chelating reagents, which could chelate with alkali metals, rare-earth elements or transition metals to form stable watersoluble coordination compounds, and they have been widely used

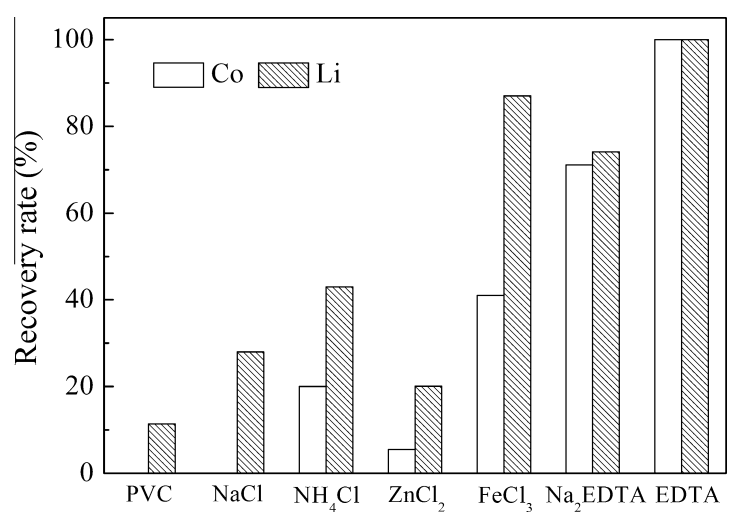

Fig. 2. Co and Li recovery rates influenced by different co-grinding reagents $\left(\mathrm{LiCoO}_{2}\right.$ to chlorides mass ratio $1: 5$, milling time $24 \mathrm{~h}$, rotary speed $600 \mathrm{rpm}$, ballto-powder mass ratio 125:1.) for the removal of heavy metals from industrial waste (Nowack, 2002; Sasai et al., 2008). Accordingly, it can be concluded that both $\mathrm{Na}_{2}$ EDTA and EDTA could react with $\mathrm{LiCoO}_{2}$ to form water-soluble metal chelates, and thereby to recover $\mathrm{Co}$ and Li by solid-solid reaction. Meanwhile, owing to the presence of carboxyl, EDTA outperformed than $\mathrm{Na}_{2}$ EDTA in chelate with $\mathrm{Co}$ and Li. The relevant working mechanism of $\mathrm{LiCoO}_{2}$ co-grinded with EDTA will be further discussed.

\subsection{Effects of various parameters on recovery rates of Co and $L i$}

The effects of $\mathrm{LiCoO}_{2}$ to EDTA mass ratio on the recovery rates of $\mathrm{Co}$ and $\mathrm{Li}$ are shown in Fig. 3a. Both recovery rates of $\mathrm{Co}$ and $\mathrm{Li}$ increased rapidly along with the decrease of mass ratio, e.g., $26 \%$ for Co and $44 \%$ for Li were recovered at mass ratio $1: 1$, while $98 \%$ of $\mathrm{Co}$ and $99 \%$ of Li were recovered at mass ratio 1:4. Then recovery rates almost unchanged when the mass ratio were $1: 5$ and $1: 6$. The above results implied that 1:4 was the maximum mass ratio for the recovery process. This is consistent with a previous report, showing that EDTA generally coordinates with metal ions at the molar ratio of $1: 1$, with the $\mathrm{LiCoO}_{2}$ to EDTA mass ratio of 1:3.6 (Wang et al., 2012).

Fig. $3 \mathrm{~b}$ indicates that the recovery rates of $\mathrm{Co}$ and $\mathrm{Li}$ increased with the prolonging of ball milling time. The recovery rates increased significantly from $67 \%$ to $89 \%$ for Co and $66-90 \%$ for $\mathrm{Li}$, respectively, as the ball milling time was prolonged from $1 \mathrm{~h}$ to $3 \mathrm{~h}$. After ball milling treatment of $4 \mathrm{~h}$, the recovery rates of Co and $\mathrm{Li}$ reached $98 \%$ and $99 \%$, respectively, then the recovery rates remained almost the same at $4.5 \mathrm{~h}$ and $5 \mathrm{~h}$ ball milling time. It has been proved that prolonging reaction time would break down the crystal structure of ball milled powder thoroughly and provide more energy to the system (Balaz et al., 2013). Therefore, the recovery rates of both $\mathrm{Co}$ and $\mathrm{Li}$ increased with the grinding degree of sample powders. Considering the energy consumption and recovery rates, it is recommended that $4 \mathrm{~h}$ is the optimum milling time.

As reported, the mode of grinding medium on the material way and the magnitude of the force depend on the milling speed, which means that a higher speed provides larger force to totally destroy the material particles (Nasser and Mingelgrin, 2012). The effects of ball milling rotary speed on recovery rates of $\mathrm{Co}$ and $\mathrm{Li}$ are shown in Fig. 3c. The recovery rates increased sharply from $38 \%$ to $90 \%$ for Co and $42-97 \%$ for $\mathrm{Li}$, respectively, as the rotary speed increased from $300 \mathrm{r} / \mathrm{min}$ to $500 \mathrm{r} / \mathrm{min}$. Then, the recovery rates of Co and Li reached maximum values of $98 \%$ and $99 \%$ at the ball milling rotary speed of $600 \mathrm{r} / \mathrm{min}$. Hence $600 \mathrm{r} / \mathrm{min}$ was the suitable rotary speed for Co and Li recovery.

Ball-to-powder mass ratio is a specific value of grinding medium quality and material quality during ball milling treatment. It also plays an important role in the ball milling process as inappropriate ball-to-powder mass ratio will increase the unnecessary energy loss and weaken the ball milling ability (Guo et al., 2010). Fig. $3 \mathrm{~d}$ shows the effects of ball-to-powder mass ratio on the recovery of $\mathrm{Co}$ and $\mathrm{Li}$. The results indicated that the recovery rates of Co and $\mathrm{Li}$ both enhanced with the increase of ball-to-powder mass ratio. The recovery rate of $\mathrm{Co}$ and $\mathrm{Li}$ reached maximum values of 97\% and $99 \%$ for Co and Li respectively at a ball-to-powder mass ratio of 80:1. To summarize, the optimum conditions of $\mathrm{Co}$ and $\mathrm{Li}$ recovery from spent LIBs were: $\mathrm{LiCoO}_{2}$ to EDTA mass ratio 1:4, milling time $4 \mathrm{~h}$, rotary speed $600 \mathrm{r} / \mathrm{min}$ and ball-to-powder mass ratio 80:1, respectively. Further separation of Co and Li could be achieved by addition of sodium hydroxide and sodium carbonate solutions. 


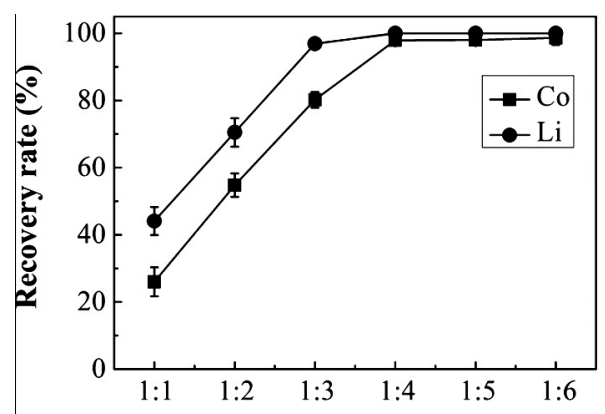

(a) Mass ratio of $\mathrm{LiCoO}_{2}$ to EDTA

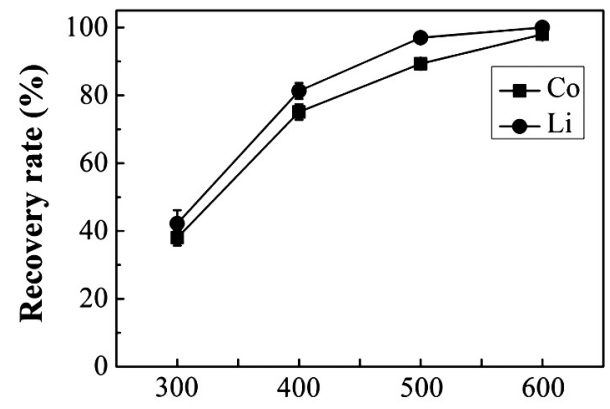

(c) Rotary speed (r/min)

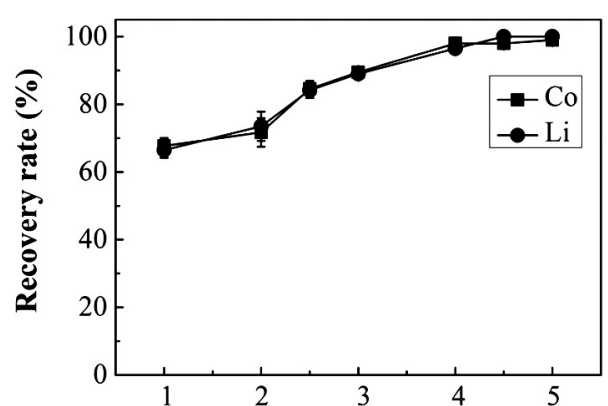

(b) Milling time (h)

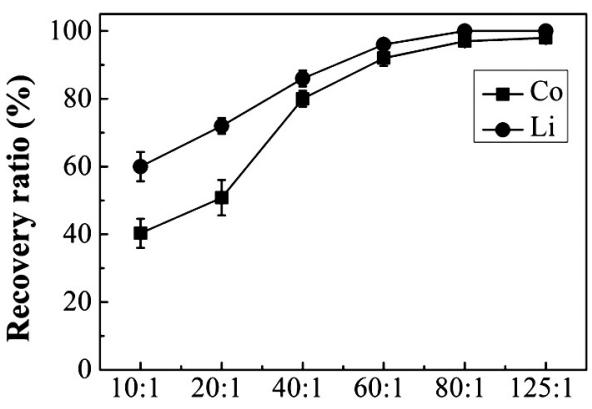

(d) Ball-to-powder mass ratio

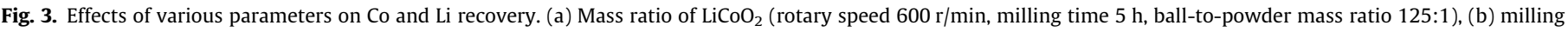

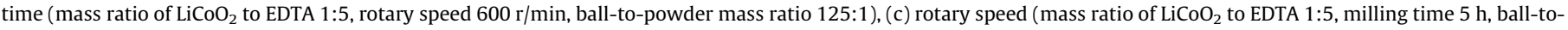
powder mass ratio 125:1) and (d) ball-to-powder mass ratio (mass ratio of $\mathrm{LiCoO}_{2}$ to EDTA 1:5, milling time $5 \mathrm{~h}$ and rotary speed $600 \mathrm{r} / \mathrm{min}$ ).

\subsection{Reaction mechanism of $\mathrm{LiCOO}_{2}$ with EDTA during ball milling}

$\mathrm{LiCoO}_{2}$-EDTA ball milling process under the influence of mechanochemical treatment can be divided into two stages, i.e. enhancement of reactant activity and diffusion of reactant into water. In general, the reactant activity of raw material could be promoted by mechanical force. The crystal lattice structure of reactant could be destroyed, inducing the reorganization and distortion of the lattice when reactant is subjected to external forces in the confined system (Li et al., 2010b; Yuan et al., 2012; Yuan et al., 2013a,b).

During ball milling progress, the dispersal mixture of $\mathrm{LiCoO}_{2}$ and EDTA was grinded evenly. The crystal grain was being tiniest along with collision with high speed grinding balls and the steel tank wall, which caused the rapid increase of specific surface area and energy. Accordingly, the crystal lattice of $\mathrm{LiCoO}_{2}$ and EDTA generated lattice distortion and local damage, and formed surface defects and high density dislocation, which resulting the increase of internal energy and the enhancement of reactant activity. Fig. 4a clearly identified the reflections of EDTA and $\mathrm{LiCoO}_{2}$ phase, while in Fig. 4b, no reflections of EDTA was observed and the reflections of $\mathrm{LiCoO}_{2}$ became weaker after ball milling treatment. The inner energy of crystal lattice was enhanced after mechanical activation treatment, which was feasible for atomic transition (Kaupp, 2009). In fact, the following water leaching process was a disordering diffusion process for the activated material in water. The diffusion was intensified along with the decrease of diffusion activation energy through ball milling, which realized the rapid leaching for reactants at low temperature.

The distance between reactants was shortened by the friction and wear performance between powders, furthermore, atomic spontaneous diffusion rate was directly increased by mechanical force as well, then chemical reaction was induced between EDTA and $\mathrm{LiCoO}_{2}$ (Wu et al., 2013; Yang et al., 2015). The chelate reaction mechanism of EDTA- $\mathrm{LiCoO}_{2}$ milling is shown below:<smiles>O=C(O)CN(CCN(CC(=O)O)CC(=O)O)CC(=O)O</smiles>

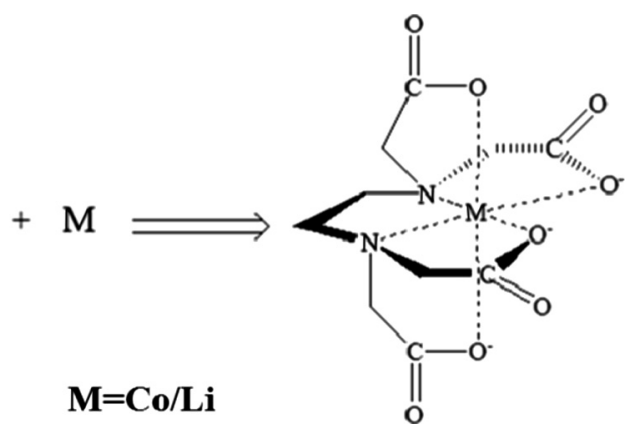




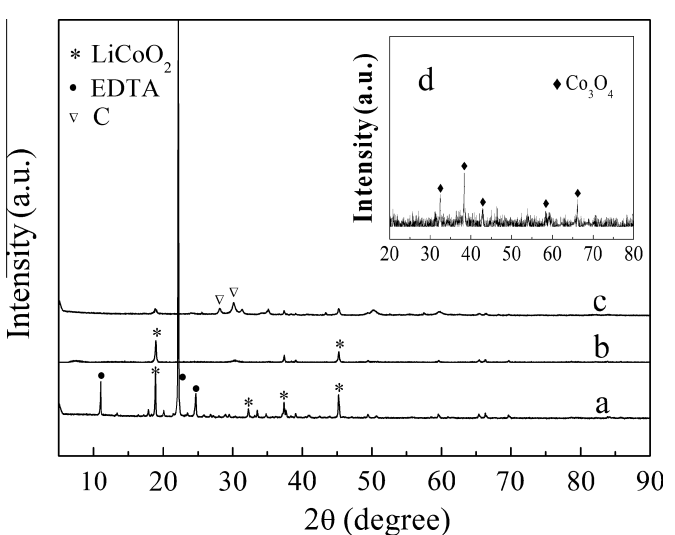

Fig. 4. XRD patterns of different samples: (a) $\mathrm{LiCoO}_{2}$ and EDTA mixture before ball milling, (b) $\mathrm{LiCoO}_{2}$ and EDTA mixture after ball milling, (c) water leaching residues after ball milling. Conditions: $\mathrm{LiCoO}_{2}$ to EDTA mass ratio $1: 5$, milling rotary speed $600 \mathrm{r} / \mathrm{min}$, milling time $5 \mathrm{~h}$, ball-to-powder mass ratio 125:1 and (d) XRD pattern of recovered $\mathrm{Co}_{3} \mathrm{O}_{4}$ particle.

It has been reported that metal chelates are cyclic structure of metal atoms and organic ligand formed by donation of electrons from the ligand donor atoms, usually oxygen, nitrogen, or sulfur is the electron acceptor metal ion. The chemical bond formed in this manner may vary in characteristics from the covalent to the almost completely ionic type (Rubin and Princiotto, 1963). EDTA, which is an aminopolycarboxylic acid containing six ligands, two amines and four carboxyl groups, can coordinate with metal ions at the molar ratio of $1: 1$. During the ball milling process, lone pair electrons provided by nitrogen oxygen atoms of EDTA could enter the empty orbit of Co and Li by solid-solid reaction, forming stable and water-soluble metal complexes Li-EDTA (stability constant lg $\mathrm{K}=2.79$ ) and Co-EDTA (stability constant $\lg \mathrm{K}=36.0$ ), respectively. The XRD pattern of recovered particle was shown in Fig. 4d, where the reflections of $\mathrm{Co}_{3} \mathrm{O}_{4}$ phase were clearly identified. Furthermore, ICP-OES result showed about $94 \%$ cobalt was recovered in the form of $\mathrm{CO}_{3} \mathrm{O}_{4}$.

\section{Conclusions}

A high efficiency and environmentally friendly mechanochemical process with EDTA as metal chelate reagent was established for Co and Li recovery from spent LIBs. The process includes 3 procedures: (1) co-grinding $\mathrm{LiCoO}_{2}$ powder with EDTA, (2) leaching the ball milled mixture with water, and (3) recovery of Co and $\mathrm{Li}$ in the form of $\mathrm{CO}_{3} \mathrm{O}_{4}$ and $\mathrm{Li}_{2} \mathrm{CO}_{3}$ by chemical precipitation. The optimal mechanochemical conditions were: $\mathrm{LiCoO}_{2}$ to EDTA mass ratio $1: 4$, milling time $4 \mathrm{~h}$, rotary speed $600 \mathrm{r} / \mathrm{min}$ and ball-to-powder mass ratio $80: 1$, respectively, under which $98 \%$ of Co and $99 \%$ of $\mathrm{Li}$ could be recovered. It is believed that the developed process is efficient and practical for materials recycling from spent LIBs, and furthermore, this process is feasible to scale up the reduction of environmental loads resulting from spent LIBs recycling.

\section{Acknowledgements}

This research was financially supported by the National Natural Science Foundation of China (21477151, 51278485), the Strategic Priority Research Program of the Chinese Academy of Sciences (XDB05050200) and the Science Promotion Program of Research Center for Eco-Environmental Sciences, CAS (YSW2013B05).

\section{References}

Al-Thyabat, S., Nakamura, T., Shibata, E., Iizuka, A., 2013. Adaptation of minerals processing operations for lithium-ion (LiBs) and nickel metal hydride (NiMH) batteries recycling: critical review. Miner. Eng. 45, 4-17.

Balaz, P., Achimovicova, M., Balaz, M., Billik, P., Cherkezova-Zheleva, Z., Criado, J.M., Delogu, F., Dutkova, E., Gaffet, E., Gotor, F.J., Kumar, R., Mitov, I., Rojac, T., Senna, M., Streletskii, A., Wieczorek-Ciurowa, K., 2013. Hallmarks of mechanochemistry: from nanoparticles to technology. Chem. Soc. Rev. 42, $7571-7637$.

Bertuol, D.A., Toniasso, C., Jiménez, B.M., Meili, L., Dotto, G.L., Tanabe, E.H., Aguiar, M.L., 2015. Application of spouted bed elutriation in the recycling of lithium ion batteries. J. Power Sources 275, 627-632.

Chaudhary, A., Donaldson, J., Grimes, S., Yasri, N., 2000. Separation of nickel from cobalt using electrodialysis in the presence of EDTA. J. Appl. Electrochem. 30, 439-445.

Chen, L., Tang, X., Zhang, Y., Li, L., Zeng, Z., Zhang, Y., 2011. Process for the recovery of cobalt oxalate from spent lithium-ion batteries. Hydrometallurgy 108, 80-86.

Chen, X., Chen, Y., Zhou, T., Liu, D., Hu, H., Fan, S., 2015. Hydrometallurgical recovery of metal values from sulfuric acid leaching liquor of spent lithium-ion batteries. Waste Manage. 38, 349-356.

Courtney, I.A., Dahn, J., 1997. Electrochemical and in situ X-ray diffraction studies of the reaction of lithium with tin oxide composites. J. Electrochem. Soc. 144, 2045-2052.

Directive, B., 2006. Directive 2006/66/EC of the European Parliament and of the Council of 6 September 2006 on batteries and accumulators and waste batteries and accumulators and repealing Directive 91/157/EEC. Off. J. Eur. Union L 266

Directive, C., 1991. 91/157/EEC on batteries and accumulators containing certain dangerous substances. Off. J. Eur. Union L 78, 38-41.

Directive, C., 2008. Directive 2008/103/EC of the European Parliament and of the Council of 19 November 2008 Amending Directive 2006/66/EC on batteries and accumulators and waste batteries and accumulators as regards placing batteries and accumulators on the market. Off. J. Eur. Union L 327.

Friščić, T., 2010. New opportunities for materials synthesis using mechanochemistry. J. Mater. Chem. 20, 7599.

Guo, X., Xiang, D., Duan, G., Mou, P., 2010. A review of mechanochemistry applications in waste management. Waste Manage. 30, 4-10.

Iizuka, A. Yamashita, Y. Nagasawa, H., Yamasaki, A., Yanagisawa, Y. 2013. Separation of lithium and cobalt from waste lithium-ion batteries via bipolar membrane electrodialysis coupled with chelation. Sep. Purif. Technol. 113, 3341.

James, S.L., Adams, C.J., Bolm, C., Braga, D., Collier, P., Friscic, T., Grepioni, F., Harris, K.D., Hyett, G., Jones, W., Krebs, A., Mack, J., Maini, L., Orpen, A.G., Parkin, I.P., Shearouse, W.C., Steed, J.W., Waddell, D.C., 2012. Mechanochemistry: opportunities for new and cleaner synthesis. Chem. Soc. Rev, 41, 413-447.

Jha, M.K., Kumari, A., Jha, A.K., Kumar, V., Hait, J., Pandey, B.D., 2013. Recovery of lithium and cobalt from waste lithium ion batteries of mobile phone. Waste Manage. 33, 1890-1897.

Joulié, M., Laucournet, R., Billy, E., 2014. Hydrometallurgical process for the recovery of high value metals from spent lithium nickel cobalt aluminum oxide based lithium-ion batteries. J. Power Sources 247, 551-555.

Kang, D.H., Chen, M., Ogunseitan, O.A., 2013. Potential environmental and human health impacts of rechargeable lithium batteries in electronic waste. Environ. Sci. Technol. 47, 5495-5503.

Kano, J., Kobayashi, E., Tongamp, W., Miyagi, S., Saito, F., 2009. Non-thermal reduction of indium oxide and indium tin oxide by mechanochemical method. J. Alloy. Compd. 484, 422-425.

Kaupp, G., 2009. Mechanochemistry: the varied applications of mechanical bondbreaking. CrystEngComm 11, 388-403.

Lee, C.-H., Jeong, M.-K., Fatih Kilicaslan, M., Lee, J.-H., Hong, H.-S., Hong, S.-J., 2013. Recovery of indium from used LCD panel by a time efficient and environmentally sound method assisted HEBM. Waste Manage. 33, 730-734.

Li, L., Ge, J., Wu, F., Chen, R., Chen, S., Wu, B., 2010a. Recovery of cobalt and lithium from spent lithium ion batteries using organic citric acid as leachant. J. Hazard. Mater. 176, 288-293.

Li, L, Ou, W Z, Zhang X, Lu, J. Chen, R.,Wu, F, Amine, K, 2015. Succinic acid-based leaching system: a sustainable process for recovery of valuable metals from spent Li-ion batteries. J. Power Sources 282, 544-551.

Li, M.-G., Sun, C.-J., Gau, S.-H., Chuang, C.-J., 2010b. Effects of wet ball milling on lead stabilization and particle size variation in municipal solid waste incinerator fly ash. J. Hazard. Mater. 174, 586-591.

Majeau-Bettez, G., Hawkins, T.R., Stromman, A.H., 2011. Life cycle environmental assessment of lithium-ion and nickel metal hydride batteries for plug-in hybrid and battery electric vehicles. Environ. Sci. Technol. 45, 4548-4554.

Nasser, A., Mingelgrin, U., 2012. Mechanochemistry: a review of surface reactions and environmental applications. Appl. Clay Sci. 67-68, 141-150.

Nayaka, G.P., Pai, K.V., Santhosh, G., Manjanna, J., 2016. Dissolution of cathode active material of spent Li-ion batteries using tartaric acid and ascorbic acid mixture to recover Co. Hydrometallurgy 161, 54-57.

Notter, D.A., Gauch, M., Widmer, R., Wager, P., Stamp, A., Zah, R., Althaus, H.-J.r. 2010. Contribution of Li-ion batteries to the environmental impact of electric vehicles. Environ. Sci. Technol. 44, 6550-6556.

Nowack, B., 2002. Environmental chemistry of aminopolycarboxylate chelating agents. Environ. Sci. Technol. 36, 4009-4016. 
Paulino, J.F., Busnardo, N.G., Afonso, J.C., 2008. Recovery of valuable elements from spent Li-batteries. J. Hazard. Mater. 150, 843-849.

Provazi, K., Campos, B.A., Espinosa, D.C.R., Tenório, J.A.S., 2011. Metal separation from mixed types of batteries using selective precipitation and liquid-liquid extraction techniques. Waste Manage. 31, 59-64.

Rubin, M., Princiotto, J., 1963. Chelation in nutrition, chelation as a basic biological mechanism. J. Agric. Food Chem. 11, 98-103.

Saeki, S., Lee, J., Zhang, Q., Saito, F., 2004. Co-grinding $\mathrm{LiCoO}_{2}$ with PVC and water leaching of metal chlorides formed in ground product. Int. J. Miner. Process. 74, S373-S378.

Sasai, R., Kubo, H., Kamiya, M., Itoh, H., 2008. Development of an eco-friendly material recycling process for spent lead glass using a mechanochemical process and $\mathrm{Na}_{2}$ EDTA reagent. Environ. Sci. Technol. 42, 4159-4164.

Scrosati, B., Garche, J., 2010. Lithium batteries: status, prospects and future. J. Power Sources 195, 2419-2430.

Shibata, J., Murayama, N., Niinae, M., Furuyama, T., 2011. Environmentally friendly separation and recovery process of tungsten and cobalt from tungsten carbide tool waste. J. Jpn. Inst. Met. 75, 613-619.

Shibata, J., Murayama, N., Niinae, M., Furuyama, T., 2012. Development of advanced separation technology of rare metals using extraction and crystallization stripping. Mater. Trans. 53, 2181-2186.

Sun, L., Qiu, K., 2011. Vacuum pyrolysis and hydrometallurgical process for the recovery of valuable metals from spent lithium-ion batteries. J. Hazard. Mater. $194,378-384$

Sun, L., Qiu, K., 2012. Organic oxalate as leachant and precipitant for the recovery of valuable metals from spent lithium-ion batteries. Waste Manage. 32, 15751582.

Tan, Q., Li, J., 2015. Recycling metals from wastes: a novel application of mechanochemistry. Environ. Sci. Technol. 49, 5849-5861.

Wang, H., Jang, Y.I., Huang, B., Sadoway, D.R., Chiang, Y.M., 1999. TEM study of electrochemical cycling-induced damage and disorder in $\mathrm{LiCoO}_{2}$ cathodes for rechargeable lithium batteries. J. Electrochem. Soc. 146, 473-480.

Wang, M., Wang, N., Tang, H., Cao, M., She, Y., Zhu, L., 2012. Surface modification of nano- $\mathrm{Fe}_{3} \mathrm{O}_{4}$ with EDTA and its use in $\mathrm{H}_{2} \mathrm{O}_{2}$ activation for removing organic pollutants. Catal. Sci. Technol. 2, 187-194.
Wang, R.-C., Lin, Y.-C., Wu, S.-H., 2009. A novel recovery process of metal values from the cathode active materials of the lithium-ion secondary batteries. Hydrometallurgy 99, 194-201.

Wu, C.-W., Sun, C.-J., Gau, S.-H., Hong, C.-L., Chen, C.-G., 2013. Mechanochemically induced synthesis of anorthite in MSWI fly ash with kaolin. J. Hazard. Mater. 244-245, 412-420.

Xu, J., Thomas, H.R., Francis, R.W., Lum, K.R., Wang, J., Liang, B., 2008. A review of processes and technologies for the recycling of lithium-ion secondary batteries. J. Power Sources 177, 512-527.

Yang, Y., Zhang, S., Wang, S., Zhang, K., Wang, H., Huang, J., Deng, S., Wang, B., Wang, Y., Yu, G., 2015. Ball milling synthesized MnOx as highly active catalyst for gaseous POPs removal: significance of mechanochemically induced oxygen vacancies. Environ. Sci. Technol. 49, 4473-4480.

Yuan, W., Li, J., Zhang, Q., Saito, F., 2012. Innovated application of mechanical activation to separate lead from scrap cathode ray tube funnel glass. Environ. Sci. Technol. 46, 4109-4114.

Yuan, W., Li, J., Zhang, Q., Saito, F., Yang, B., 2013a. Lead recovery from cathode ray tube funnel glass with mechanical activation. J. Air Waste Manage. Assoc. 63, 2 10.

Yuan, W., Li, J., Zhang, Q., Saito, F., Yang, B., 2013b. A novel process utilizing mechanochemical sulfidization to remove lead from cathode ray tube funnel glass. J. Air Waste Manage. Assoc. 63, 418-423.

Zeng, X., Li, J., Ren, Y., 2012. Prediction of various discarded lithium batteries in China. In: IEEE International Symposium on Sustainable Systems and Technology (ISSST), 2012. IEEE, pp. 1-4.

Zeng, X., Li, J., Shen, B., 2015. Novel approach to recover cobalt and lithium from spent lithium-ion battery using oxalic acid. J. Hazard. Mater. 295, 112-118.

Zeng, X., Li, J., Singh, N., 2014. Recycling of spent lithium-ion battery: a critical review. Crit. Rev. Environ. Sci. Technol. 44, 1129-1165.

Zhang, Q., Saeki, S., Tanaka, Y., Kano, J., Saito, F., 2007. A soft-solution process for recovering rare metals from metal/alloy-wastes by grinding and washing with water. J. Hazard. Mater. 139, 438-442.

Zhang T, He, Y, Ge, L, Fu, R., Zhang, X., Huang Y, 2013. Characteristics of wet and dry crushing methods in the recycling process of spent lithium-ion batteries. J. Power Sources 240, 766-771. 